\title{
Role of Machine Learning and Big Data in Progress of Smart City
}

\author{
Hiba Iftikhar ${ }^{1}$, Ranjit Biswas ${ }^{2}$ \\ \{hiba.iftikhar14@gmail.com ${ }^{1}$, ranjitbiswas@yahoo.com ${ }^{2}$ \} \\ Department of Computer Science Engineering Jamia Hamdard University, Delhi, India
}

\begin{abstract}
There are lot of problems in implementing smart cities because of large number of people with diverse and varied prerequisite. This paper offers and studies the angles and impact of big data and machine learning as approach for smart cities organization and growth. The problems of smart cities cannot be solved without the consideration of Big data in smart cities but due to lack of mechanism and knowledge most of the data is wasted and the benefit which could have been derived from the data are lost. This study aims review applications of big data and machine learning to support smart cities. It finds the advantages in considering of big data and machine learning in developing of smart cities and in understanding its role and its challenges. This paper reviews that there are various chances for the collection and utilization of big data collected from smart cities but there are also challenges in using this data for the betterment of the cities.
\end{abstract}

Keywords: Big Data, Internet of Things, Machine Learning, Smart City.

\section{Introduction}

According to the United Nations Population Division, urban regions only cover 0.2 per cent of Earth's land surface but contain nearly half of the world's population.t puts extreme pressure on today's cities and resulting in the generation of large quantities of data at unprecedented rates. Reports of the UN estimated that the population growth rate in urban areas would hit $68 \%$ by 2050. [1]

Moreover, $70 \%$ of the world's resources are consumed by cities. Many governments want to convert their city to smart city by correcting the data and automating this system so that they can provide better facilities with unique ideas to develop their cities and provide intelligent and economical solutions to their complex problems of providing civic enmities and raised their living standard of their people.

The problems of mostly all the cities are increases in traffic, population limited resources, waste and waste management and providing electricity to everyone and many more. Because due to insufficient mechanisms and standards most of the collected data which could be useful to provide knowledge and solution to solve the problems of the city gets wasted. 


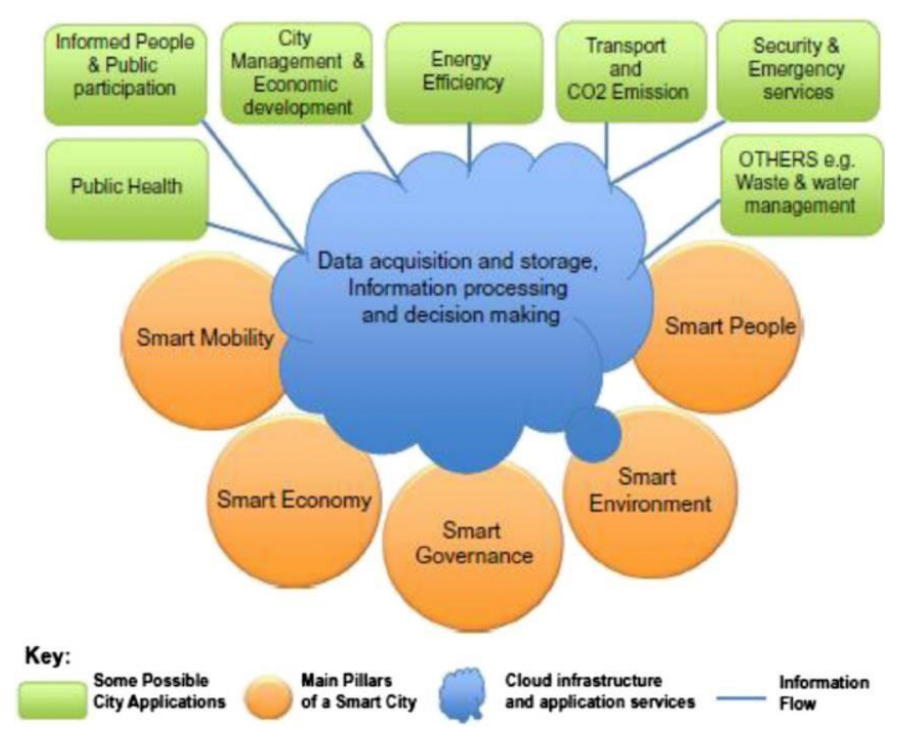

Fig.1. The closed diagram generated by collected data [10]

\section{Literature Review}

The main aim of the smart cities is to control the existing resources carefully, sustainably, and professionally. System, people and things in the urban areas generates data. Internet of things (IOT) is the sensors which are used by the cities to collect data and then use this data to utilize resources in efficient manner. This collected data is obtained from people, devices, and resources, then it is handled and studied so that we can achieve our goal of smart cities by managing water supply, waste management, traffic, hospitals, schools etc.

The data collected is the most important raw material for the development of smart cities. However due to very diverse nature of data it is difficult to organize, translate, recognize and consume. Data is enormously big and it comes from very different resources like, building, environment, energy, water so large storage and computation facilities are required. 


\section{Big Data Description}

Data is very important to achieve the goal of smart cities, we require dependable data so that we can rely on it and make our future decisions based on the calculation and processing of data received.

\subsection{Stages of Data}

- In the first stage the data collected through IOT and the sensors which are placed in the cities are raw data. Devices used to collect data are connected to high speed communication networks.

- In the second stage the raw data collected in first stage is calculated and analyzed by using applications and software provides by technology developers.

- In the third stage it is necessary for the education of citizens so that every citizen should be aware of the technology and could use and adopt it.

Third step:

Adoption and usage for citizens leading to better changes and behavior

Second step:

Smart applications and data-analysis capabilities

The Tech Base:

Includes networks of connected devices and sensors to collect data

Fig .2. Three stages of Data [13] 


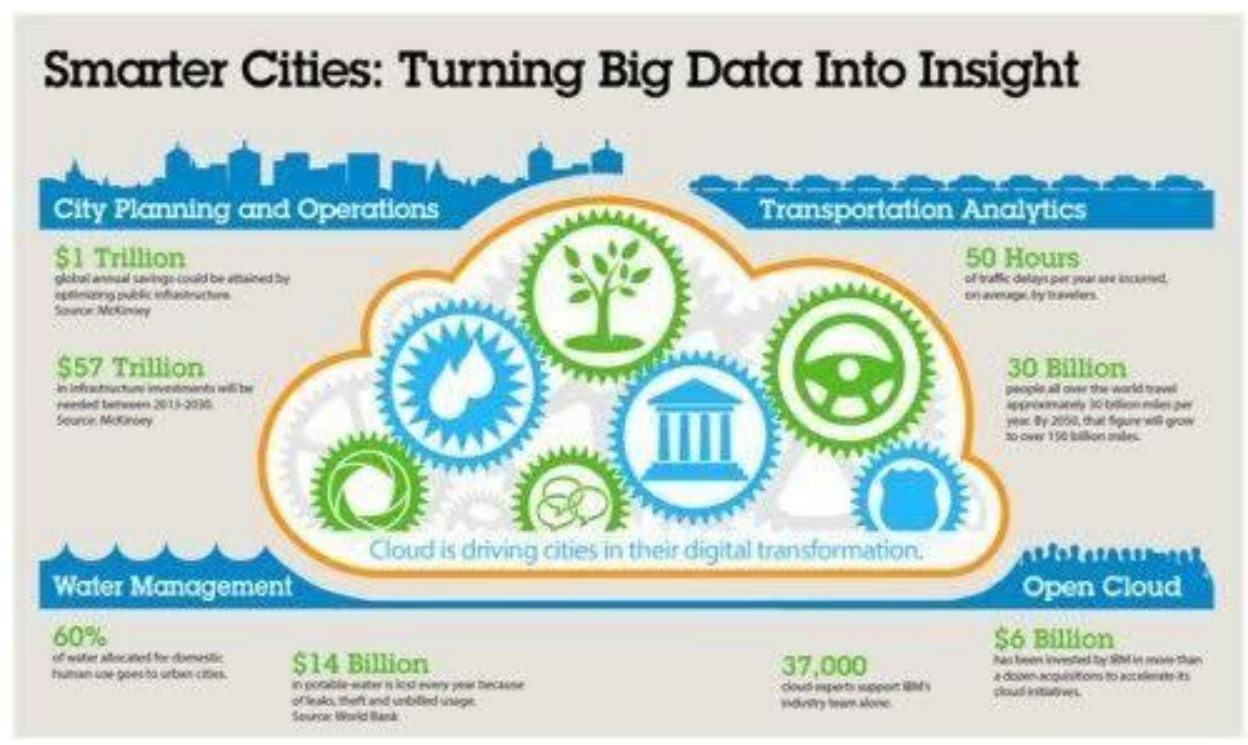

Fig.3. Big data impact on smart cities [13]

\section{Data Challenges and Solutions}

There is enormous amount of data collected in the cities, but it could not be fully used for the development of cities because of its limited access. These data include the government maps, statistics, tenders which are used by the government for specific goal, but it should be used to achieve common good i.e., smart cities.

Better pollution control, lightening, waste management, education, disaster planning are places where technology can make a huge impact. However, there are lot of disintegration we need to collect it and bring to common platform.

Development of smart cities require corporation not only by public sector but also by private sector. Data collected should be shared between the sectors so that it can be analyzed to support automation which in turn help in making the cities smarter.

Big data which is collected through various sources is enormous in size and stored at various sites and most of it is not used. So, there is requirement of better understanding of stored data by using technology in fields of data mining, data intelligence and data analytics. The Cloud system could be the solution because it can be a platform for resource applications to share and collaborate the data between applications. To achieve the goal of smarter cities Cloud platform can perform efficient role and solve some of the problems faced in developing smart cities.

The efficient management of data is required. There can be huge data which is produced every second. The data is captured, stored, and then not analyzed completely. Smart 
cities require achieving free communication of data through networks.

\section{Collection and Storage of Data}

\subsection{Information and Communications Technology (ICT)}

Information and communications technology (ICT) required for the development of smart cities by collecting the data from various places and making it available by using technology. This technology is called the Internet of Things (IoT), It helps in communicating between devices and sharing data that requires wireless connections, internet, and other communication mediums.

Here are various IoT devices placed around the city whose main job is to capture data and analyze it for making user of it for that area. IoT devices collect data from different gateways installed in city and process it for the best decision making.

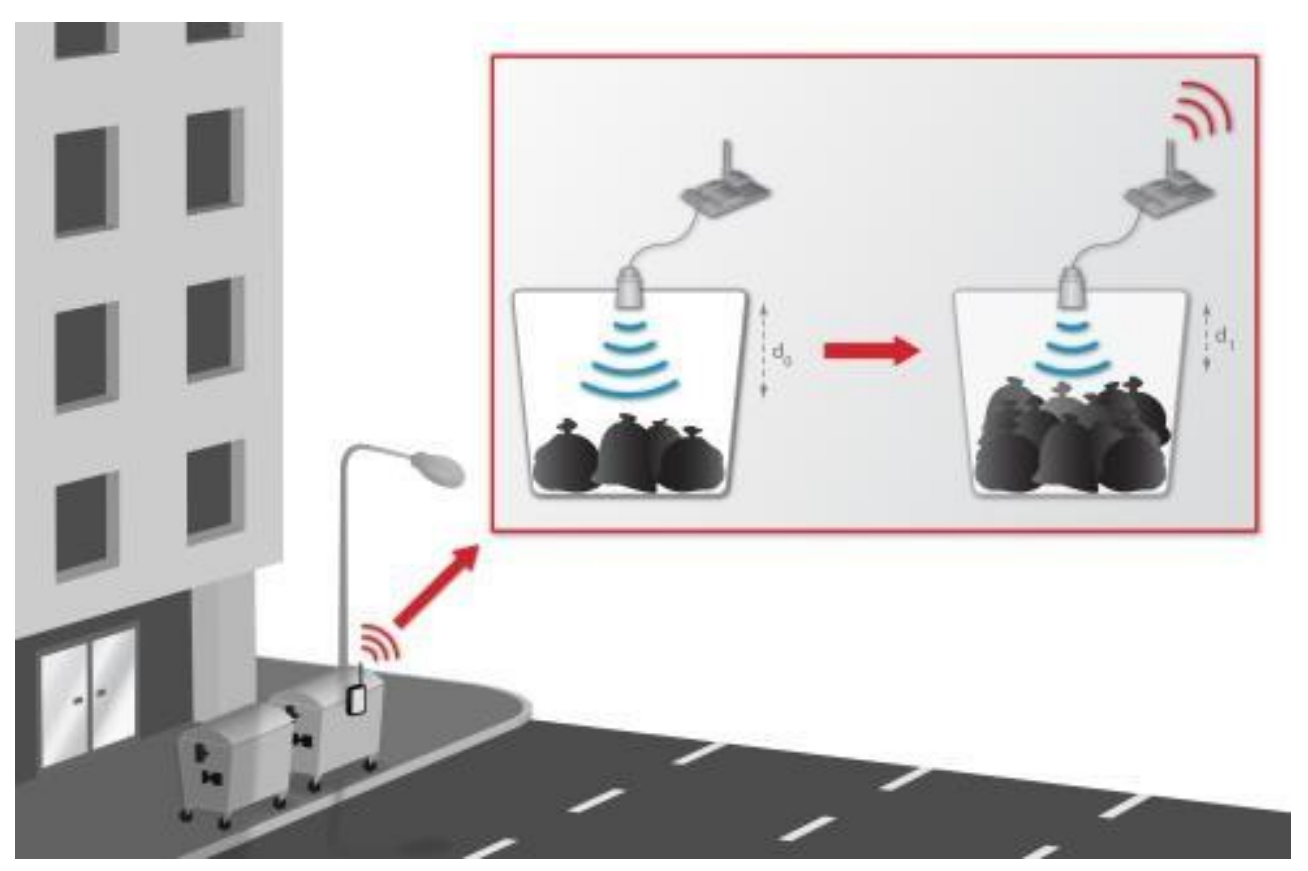

Fig.4. IOT used in city management [15]

\subsection{Cloud Computing}

Smart cities are the future of the cities which could be achieved by getting the information about the requirement of the city through use of sensors, devices, by help of technology. This data collected is stored, shared analyzed between different platforms with 
the help of technology as Cloud computing. Smart cities analyze the requirement of the city through better communication between citizens and government and quickly help in solving the problems arise in public safety, city services, and make the city run more effectively.

Smart cities imply collecting data, information from all the information providers and make it into bigger information system with the help of cloud computing and internet of things. The problem in big data is that each department collect data, but the data is not used properly due to the lack of communication between different departments. The sharing mechanism is also not working systematically. So we require unified standards for data to be communicated and shared between different departments. By achieving this we can make a system for massive data sharing and provide intelligent services and achieve smart city objective.

To achieve smart city the requirement of better communication infrastructure ,construction of efficient information platform, and make the citizens more aware of the technology so that they can receive intelligent services .A smart city should have city information cloud which in cooperate all city services on a single platforms it becomes easy and efficient in sharing information between different departments and provide intelligent services by help of technology .The smart city should have infrastructure cloud, platform cloud and application cloud. The infrastructure cloud compromises server, storage and network. It is the backbone as it handles the captured data and its storage through high speed and efficient communication networks. The platform cloud main job is to take the data from different infrastructure clouds and share the data by means of data switching and data collection and perform mathematical computation for better use of data. The main purpose of application cloud is implementation platform. In it we can construct government services related clouds to meet the requirements from city activities.

\section{Benefits and Opportunities}

The data captured from smart cities are not consistent and due to its changing nature, we require machine learning techniques which get intelligent and learn from real time data and perform necessary analysis of data. There are few examples of machine learning applications in smart cities such as:

\subsection{Smart Street Lighting}

Smart street lightning compromises of replacing old fashioned bulbs with new LED bulbs which reduces the carbon emissions, and are more efficient and have longer life, which results in decrease in cost for smart cities. There is well connected infrastructure which could monitor and maintain when any sort of problem arises.

\subsection{Intelligent Parking}

Major cause of traffic in cities are caused by drivers looking for vacant place for parking and resulting in increasing amount of carbon emission and putting strain on traffic. Intelligent parking help to solve the problem of parking by installing IoT devices which could 
help in to locate empty spaces for parking and guide drivers.

\subsection{Video Analyses}

Cameras are located on the streets and the recording is analyzed in real time with the help of machine learning algorithms and if there are some abnormality according to the software, it alerts the concerned department automatically. Thus, reducing criminal activities.

\section{Challenges}

\subsection{Data Formats:}

Data is capture from diverse sources in many different formats. There are many formats for raw data available in market e.g. tweet, image, audio, logs etc. There is requirement of database software which converts these different formats into structured format and stores it for better management of data.

\subsection{Data security:}

Data Security is a problem which smart cities face because as all the data is captured irrespective it is public or private and stored in cloud, it is also shared between different users . It is therefore require to protect the private data to fall in corrupt hands. By using data security methods we can safeguard private data.

\subsection{Government policies:}

There should be effort from the government to educate the use of technology to its citizens and government should allocate funds in implementing new technology and sensors in places when and where required.

\section{Requirements}

Data is captured in large amount from the sensors and users and there are problems in handling this ever-increasing amount of data, which results in requirement of storing, processing, and organizing of data to produce effective results. The other requirement is for sharing the data which is stored and better communication between all stake holders of smart cities.

\subsection{Data Management}

As we know technologies always get advanced due to which we must update our existing systems and infrastructure with respect to it. Now most of the data is live streaming from social networking sites, emails, blogs etc. even large amount of machine generated data. So, we must put in strategies which includes Stream analysis software, data correction and cleansing, data discovery, data preparation and data accessibility. 


\subsection{Effective Data-sharing}

A data sharing should be easy by using cloud. There should be consideration of the privacy of data using by digital securities the platform should support both private and public sharing.

\subsection{Citizen Awareness}

Programs to educate the use of technologies and benefits of it to the citizens to make the citizens smart and aware of the devices for better us of resources and services.

\subsection{Government Role}

Governments play an essential role in a smart city; therefore, it is required to have advanced systems to manage big data collected and used by government entities.

\section{Conclusion}

We tried to integrate the concept of machine learning and big data in achieving our goal of smart cities, therefore we discussed the applications and its requirement to get good quality of life, effective and efficient services, easy governance, and better management of the city resources. Our research is deep understanding of the concepts and their requirement and definitions. We were able to identify the requirement of technology for collection and analysis of big data and machine learning to support our development of smart cities.

We also discussed the challenges faced for development of applications for smart cities and platform for the technology which includes availability of faster communication networks to provide efficient and effective services to the users.

The application of cloud computing in smart city can solve many problems of storing data and sharing the information between different departments and can be useful in providing services in the field of education, weather, medical, transportation and logistics with better understanding of the concepts we could use the resources effectively and efficiently resulting in enhancing our cities to smart. [1] 


\section{References}

[1] https://www.nasa.gov/vision/earth/environment/urban_effects.html

[2] https://www.un.org/development/desa/en/news/population/2018-revision-of-worldurbanization- prospects.html)

[3]Wu Xibo, Yang Zaigao; the Concept of Smart City and Future City Development, Urban Studies, 17(11), 56-60 (2010)

[4] Wu Xibo, Yang Zaigao; The Concept of Smart City and Future City Development, Urban Studies, 17(11), 56-60 (2010)

[5] Gu Shengzu, Yang Jianwu, Lliu Jiangri; Problems in the Development of Smart City in China and Their Solution, China Soft Science, 1, 6-12 (2013)

[6] Bi changquan, Cao Jian, Wu Weihua, Yan Hong; Tangshan information sharing mode design based on cloud computing, Journal of Hebei University (Natural Science Edition), 32(1), 96-99 (2012).

[7] Michalik P, Stofa J, Zolotova I. Concept definition for Big Data architecture in the education system. In Applied Machine Intelligence and Informatics (SAMI), 2014 IEEE 12th International Symposium in 2014. pp. 331-334.

[8] Lee CH, Birch D, Wu C, Silva D, Tsinalis O, Li Y, Guo Y. Building a generic platform for big sensor data application. In Big Data, 2013 IEEE International Conference on IEEE; 2013. pp. 94-102.

[9] Chourabi H, Nam T, Walker S, Gil-Garcia JR, Mellouli S, Nahon K, Scholl HJ. Understanding smart cities: An integrative framework. In System Science (HICSS), 2012 45th Hawaii International Conference on IEEE; 2012. pp. 2289-2297.

[10] Bertot JC, Choi H. Big data and e-government: issues, policies, and recommendations. In Proceedings of the 14th Annual International Conference on Digital Government Research. ACM; 2013. pp. 1-10.

[11] Alexander Gonfalonieri Big Data \& Smart Cities:How Can we prepare for them https://medium.com/dataseries/big-data-and-smart-cities-why-we-need-them-nowa194b2498fb1 Dec 13, 2018

[12] Kaushik Pal Smarter Cities: Turning Big Data Into Insight https://www.kdnuggets.com/2015/10/big- data-smart-cities.html

[13] Alexandre Gonfalonieri Big Data \& Smart Cities: How Can we prepare for them https://medium.com/dataseries/big-data-and-smart-cities-why-we-need-them-nowa194b2498fb1 Dec 13, 2018. 